\title{
Quantum Theory of Magnetoelectromotive Instability in Nanoelectromechanical Systems with Positive Differential Conductance
}

\author{
D. Radić ${ }^{1,2, *}$ and L. Y. Gorelik ${ }^{2}$ \\ ${ }^{1}$ Department of Physics, Faculty of Science, University of Zagreb, 10001 Zagreb, Croatia \\ ${ }^{2}$ Department of Applied Physics, Chalmers University of Technology, SE-412 96 Gothenburg, Sweden
}

(Received 13 March 2013; published 29 October 2013)

\begin{abstract}
We consider dc-electronic transport through a nanowire suspended between two normal-metal leads in the presence of an external magnetic field. We show the very mechanism through which such a system, whose stationary current-voltage characteristic is essentially characterized by positive differential conductance, becomes unstable with respect to an onset of self-excited oscillations in electrical transport and mechanical vibrations. The self-excitation mechanism is based on the correlation between the occupancy of the quantized spin-split electronic energy levels inside the nanowire and the velocity of the nanowire with the crucial influence of strong enough retardation effects in magnetomotive coupling coming from mechanical vibrations.
\end{abstract}

DOI: 10.1103/PhysRevLett.111.186802

The physics of nanoelectromechanical systems (NEMS) and resonators at mesoscopic scales has been the central point of research in the field of nanophysics and nanotechnology for over a decade [1]. In this respect, suspended single-wall carbon nanotubes provide a unique nanosystem where both bulk beam mechanics and electronic transport exhibit pronounced mesoscopic features [2]. The central point which defines NEMS performance is an electromechanical coupling-a coupling between electronic and mechanical subsystems. One can achieve such a coupling by exploiting Coulomb or Lorentz forces induced by an external electric or magnetic field applied to the system. These forces are also used to generate mechanical oscillations if resonant ac gate or bias voltages are applied. At the same time, it is possible to generate self-sustained oscillations in NEMS by applying the dc voltage-a shuttle instability [3]. However, it requires a very special architecture of the NEMS which such simple systems as suspended carbon nanotubes do not possess. In a dc regime, Coulomb coupling between suspended nanotube and gate electrodes results only in an additional damping of mechanical oscillations [4]. In this Letter, we address a question: can we exploit Lorentz coupling to generate selfsustained oscillations of the nanotube in the dc regime? We start with an analysis of the quasiadiabatic situation when tunneling rates between source (drain) electrodes and nanotube $\Gamma_{S(D)}$ are much greater than a mechanical frequency $\omega$. There, a current through the system $I=J(V)$ is controlled by a total voltage drop $V$ between source and drain electrodes. The Lorentz force acting on the nanowire is given by $F_{L}=B L J(V)$, where $B$ is the magnetic field, $L$ is the length of the nanotube, and $V$ is a sum of the bias voltage $V_{b}$ and the electromotive force $\varepsilon \propto-B L \dot{u}$ generated by the motion of the wire in a magnetic field, where $u$ is the nanotube displacement perpendicular to $\mathbf{B}$. Therefore, for a small displacement, the Lorentz force
PACS numbers: 73.63. $-\mathrm{b}, 73.23 . \mathrm{Hk}, 75.76 .+\mathrm{j}, 85.85 .+\mathrm{j}$

takes a form $F_{L}=B L J\left(V_{b}\right)-(B L)^{2} d J /\left.d V\right|_{V_{b}} \dot{u}$, where the second term represents an effective friction generated by the interaction with an electronic subsystem. This imposes that, under the quasiadiabatic condition, the negative differential conductance (DC) produces negative friction which may result in the generation of self-sustained oscillations [5]. However, if a nanotube is suspended between normal leads, as it appears in most experimental setups, the DC is positive and magnetomotive coupling with an electronic subsystem results in the damping of mechanical oscillations in the quasiadiabatic limit. In this Letter we demonstrate the importance of the relation between tunneling rates and vibrational frequency, and show that the electromechanical instability can take place even in NEMS with positive DC if quasiadiabatic conditions are violated. This qualitatively new phenomenon is based on the correlation between electronic tunneling and the direction of velocity of the nanotube, induced by Coulomb correlations, with the crucial role of retardation coming from a mechanical subsystem.

We consider the system sketched in Fig. 1(a): the nanowire, represented as a single-level quantum dot, is suspended between two dc-biased normal-metal leads and subjected to a perpendicular external magnetic field. To describe an interplay between the nanowire vibrations and electronic transport through such a structure, we use the standard Hamiltonian [6,7]

$$
\begin{gathered}
H=H_{L}+H_{W}+H_{M}+H_{\tau}, \quad H_{L}=\sum_{\alpha, k} E_{\alpha k} a_{\alpha k}^{\dagger} a_{\alpha k}, \\
H_{W}=\sum_{\sigma} \varepsilon_{\sigma} c_{\sigma}^{\dagger} c_{\sigma}+U c_{\uparrow}^{\dagger} c_{\downarrow}^{\dagger} c_{\downarrow} c_{\uparrow}, \quad H_{M}=\frac{1}{2} \hbar \omega\left(\hat{p}^{2}+\hat{u}^{2}\right), \\
H_{\tau}=\sum_{\alpha, k, \sigma} \tau_{\alpha} v_{\alpha} c_{\sigma}^{\dagger} a_{\alpha k}+\text { H.c. }
\end{gathered}
$$


(a) nanowire

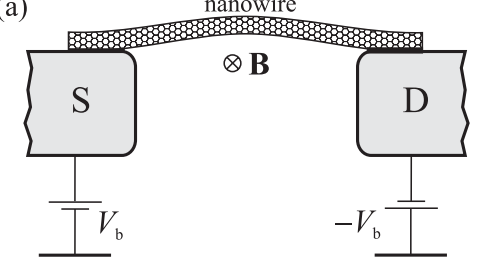

(b)

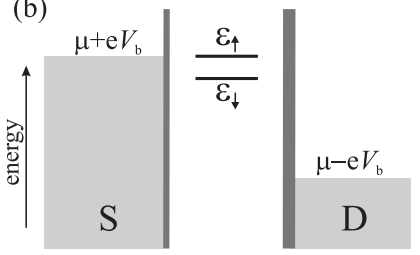

(c)

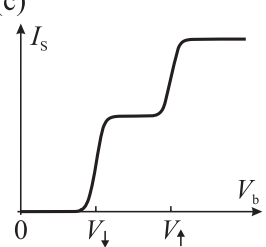

FIG. 1. (a) A nanowire subjected to an external magnetic field B, suspended between two normal-metal leads biased symmetrically by voltages $\pm V_{b}$. (b) Electronic energy scheme for the junction: $\varepsilon_{\uparrow, \downarrow}$ are spin-split levels in the nanowire, and $\mu$ is the chemical potential in the leads without bias. The junction is nonsymmetric in the sense that the tunneling barrier between $D$ and nanowire is thicker than between $S$ and nanowire. (c) A stationary current-voltage characteristic of the junction always exhibiting essentially positive differential conductance $\left(V_{\uparrow, \downarrow} \equiv \varepsilon_{\uparrow, \downarrow} / e\right)$.

$H_{L}$ describes electrons in the metal leads indexed by $\alpha=S, D$, where $a_{\alpha k}^{\dagger}$ is a creation operator for electrons with momentum $k$ and energy $E_{\alpha k}$. The electron in the nanowire is described by $H_{W}$, having two possible spinsplit states $\varepsilon_{\sigma}$, achieved, e.g., by Zeeman splitting, with corresponding creation operators $c_{\sigma}^{\dagger}, \sigma=\downarrow, \uparrow$. The second term is the Coulomb interaction $U$ between two electrons with opposite spin. Here, we assume that the spacing between quantized energy levels in the nanowire is large enough with respect to applied bias voltage $V_{b}$ so that only one pair of spin-split levels participates in the electronic transport. $H_{M}$ is the Hamiltonian describing the flexural vibration of the nanowire, which in our consideration is restricted to the dynamics of the fundamental mode only. It is treated as a harmonic oscillator with frequency $\omega$, characterized by the dimensionless displacement operator $\hat{u}$, normalized to $a_{0} \equiv \sqrt{\hbar / m \omega}$, where $m$ is the mass of the oscillator, and by the conjugated momentum $\hat{p}$ that satisfies $[\hat{u}, \hat{p}]=i$. Finally, $H_{\tau}$ describes electron tunneling between the leads and the nanowire with corresponding amplitudes $\tau_{\alpha}$. The phase factor of the tunneling amplitudes $v_{S(D)}=e^{ \pm i \lambda \hat{u}}$ depends on nanowire displacement, where the parameter $\lambda \equiv e B L a_{0} \xi / 2 \hbar c$ accounts for the magnetomotive coupling and $\xi=0.83$ is the numerical factor coming from the spatial profile of the fundamental mode [8].

To find a stationary, time independent state of the system, we start from the Liouville-von Neumann equation $[H, \varrho]=0$ for a total density matrix $\varrho$ [7]. Then, imposing that the tunneling process does not perturb states of the bulk leads significantly, we exploit the reduced density matrix approach [9], presenting the total density matrix

as a direct product $\varrho=\rho \otimes \varrho_{S} \otimes \varrho_{D}$, where $\rho$ describes the electronic and mechanical states of the nanowire, and $\varrho_{S, D}$ are the density matrices representing the source and drain electrodes as electronic reservoirs at thermal equilibrium with temperature $T$ and electrochemical potentials $\mu_{S(D)}=\mu \pm e V_{b}$. Using a standard procedure of tracing out the leads [10], one gets an equation for $\rho$ :

$$
\begin{aligned}
-i \omega[\mathcal{H}, \rho]= & \sum_{\alpha, \sigma} \int_{0}^{\infty} d z\left\{\left[v_{\alpha} U^{\dagger}(z) v_{\alpha}^{\dagger} c_{\sigma} c_{\sigma}^{\dagger} \rho U(z)\right.\right. \\
& \left.-U^{\dagger}(z) v_{\alpha}^{\dagger} c_{\sigma}^{\dagger} \rho c_{\sigma} U(z) v_{\alpha}\right] K_{\alpha \sigma}^{>}(z) \\
& +\left[U^{\dagger}(z) \rho c_{\sigma}^{\dagger} c_{\sigma} v_{\alpha}^{\dagger} U(z) v_{\alpha}\right. \\
& \left.\left.-v_{\alpha} U^{\dagger}(z) c_{\sigma} \rho c_{\sigma}^{\dagger} v_{\alpha}^{\dagger} U(z)\right] K_{\alpha \sigma}^{<}(z)+\text { H.c. }\right\},
\end{aligned}
$$

where $\mathcal{H} \equiv\left(\hat{p}^{2}+\hat{u}^{2}\right) / 2$ is the dimensionless part of $H_{M}$, $U(z) \equiv e^{i \beta \mathcal{H}_{z}}, \beta \equiv \omega / k_{B} T$, and time is put in scale with temperature, i.e., $z \equiv k_{B} T t$. The collision integral kernels are given by

$$
\begin{aligned}
K_{\alpha, \sigma}^{>,<}(z) & =\frac{1}{2} \Gamma_{\alpha}\left[\delta(z) \mp i P_{\alpha \sigma}(z)\right], \\
P_{\alpha \sigma}(z) & =\text { p.v. } \frac{e^{i\left(\mu_{\alpha}-\varepsilon_{\sigma}\right) z / k_{B} T}}{\sinh (\pi z)},
\end{aligned}
$$

where $\Gamma_{\alpha}=2 \pi \hbar^{-1} \tau_{\alpha}^{2} \nu_{\alpha}$ appears as the electron tunneling rate between the nanowire and lead $\alpha$ with electronic density of states $\nu_{\alpha}$ which is, as well as the tunneling amplitude $\tau_{\alpha}$, assumed to be independent of energy. In our setup, we choose a bias [see Fig. 1(b)] in which $\mu_{S}=$ $\varepsilon_{\uparrow}$ and require $\varepsilon_{\uparrow}-\varepsilon_{\downarrow}, \varepsilon_{\sigma}-\mu_{D} \gg k_{B} T$, meaning that the charge transfer from the $D$ lead to the nanowire, and from the $\varepsilon_{\downarrow}$ level in the nanowire to the $S$ lead, is exponentially suppressed. This simplifies kernels of collision integral greatly, namely, $P_{S \downarrow}(z) \approx i \delta(z)$ and $P_{D \sigma}(z) \approx-i \delta(z)$. Furthermore, we decompose $\rho$ into blocks describing a nanowire occupied either by spin- $\uparrow$, spin- $\downarrow$, or no electrons, $\rho=|0\rangle \rho_{0}\left\langle 0\left|+c_{\downarrow}^{\dagger}\right| 0\right\rangle \rho_{\downarrow}\left\langle 0\left|c_{\downarrow}+c_{\uparrow}^{\dagger}\right| 0\right\rangle \rho_{\uparrow}\langle 0| c_{\uparrow}$, with corresponding coefficients (matrices in mechanical space) $\rho_{\uparrow}, \rho_{\downarrow}$, and $\rho_{0}$, respectively. Imposing a Coulomb blockade constraint $U \gg e V_{b}, \varepsilon_{\sigma}-\mu$, we exclude the double occupancy of the nanowire, taking $\operatorname{Tr}\left\{c_{\uparrow}^{\dagger} c_{\downarrow}^{\dagger} c_{\uparrow} c_{\downarrow} \rho\right\}=0$, and reduce system (2) to the following set of equations:

$$
\begin{aligned}
-i \Omega\left[\mathcal{H}, \rho_{0}\right]= & \frac{3}{2} \rho_{0}-\frac{1}{2} v \rho_{\uparrow} v^{-1}-\gamma v^{-1}\left(\rho_{\uparrow}+\rho_{\downarrow}\right) v \\
& -v^{1 / 2} \widetilde{\wp}\left(\tilde{\rho}_{0}+\tilde{\rho}_{\uparrow}\right) v^{-1 / 2}, \\
-i \Omega\left[\mathcal{H}, \rho_{\uparrow}\right]= & \left(\frac{1}{2}+\gamma\right) \rho_{\uparrow}-\frac{1}{2} v^{-1} \rho_{0} v \\
& +v^{-1 / 2} \widetilde{\mho}\left(\tilde{\rho}_{0}+\tilde{\rho}_{\uparrow}\right) v^{1 / 2}, \\
-i \Omega\left[\mathcal{H}, \rho_{\downarrow}\right]= & \gamma \rho_{\downarrow}-v^{-1} \rho_{0} v,
\end{aligned}
$$


where $\quad \Omega \equiv \omega / \Gamma_{S}, \quad \gamma \equiv \Gamma_{D} / \Gamma_{S}, \quad v \equiv e^{i \lambda \hat{u}}, \quad \tilde{\rho}_{0} \equiv$ $v^{-1 / 2} \rho_{0} v^{1 / 2}, \tilde{\rho}_{\uparrow, \downarrow} \equiv v^{1 / 2} \rho_{\uparrow, \downarrow} v^{-1 / 2}$, and $\widetilde{\wp}$ is the collision integral, i.e.,

$$
\begin{aligned}
\widetilde{\Im}(\tilde{\rho})= & \frac{1}{2} \int_{0}^{\infty} d z v^{1 / 2} e^{-i \beta \mathcal{H} z} v^{-1 / 2} \tilde{\rho} v^{-1 / 2} e^{i \beta \mathcal{H} z} v^{1 / 2} P_{S \uparrow}(z) \\
& + \text { H.c. }
\end{aligned}
$$

Below, we analyze system (4) under conditions when three small parameters emerge, i.e., $\gamma, \Omega, \lambda \ll 1$, while $\beta \leq 1$. It is convenient to rewrite system (4) in Wigner representation, introducing a Wigner function of density operator $\mathbb{W}[\rho]=(1 / 2 \pi) \int d \xi e^{-i p \xi}\langle u+\xi / 2|\rho| u-\xi / 2\rangle \equiv w(u, p)$ and defining new functions $\tilde{w}_{0}=w_{0}\left(u-u_{0}, p-\lambda / 2\right)$, $\tilde{w}_{\downarrow}=w_{\downarrow}\left(u-u_{0}, p+\lambda / 2\right), \quad$ and $\quad \tilde{w}_{\uparrow}=w_{\uparrow}\left(u-u_{0}, p+\right.$ $\lambda / 2)$ which we combine into $\tilde{w}_{e} \equiv \tilde{w}_{\downarrow}+\tilde{w}_{\uparrow}$ and $\tilde{w}_{m} \equiv$ $\tilde{w}_{0}+\tilde{w}_{e}$. Displacement $u_{0}=2 \lambda \gamma / \Omega=L \bar{I} B / k a_{0}$, where $k$ is a rigidity constant and $\bar{I}$ an average current, counts the deformation of the nanowire induced by the average Lorentz force. Considering relations $\mathbb{W}[\check{\wp}(\rho)]=$ $-F(p) \mathbb{W}[\rho]+O(\lambda \beta)$, where $F(p)=(1 / 2) \tanh (\lambda \beta p / 2)$ [7], the equations for Wigner functions $\tilde{w}_{\downarrow, e, m}$, in the leading orders of small $\lambda$ accounting for both the drift and diffusion terms, are

$$
\begin{aligned}
\Omega\left\{\tilde{\mathcal{H}}, \tilde{w}_{\downarrow}\right\}= & \tilde{w}_{e}-\tilde{w}_{m}+\gamma \tilde{w}_{\downarrow} \\
\Omega\left\{\tilde{\mathcal{H}}, \tilde{w}_{e}\right\}= & (2+\gamma) \tilde{w}_{e}-\left[\frac{1}{2}-F(p)\right] \tilde{w}_{\downarrow} \\
& -\left[\frac{3}{2}+F(p)\right] \tilde{w}_{m}, \\
\Omega\left\{\tilde{\mathcal{H}}, \tilde{w}_{m}\right\}= & -2 \gamma \lambda\left\{u, \tilde{w}_{e}\right\}+\lambda \Omega\left\{p, \tilde{w}_{e}-\tilde{w}_{m}\right\} \\
& -2 \gamma \lambda^{2}\left\{u,\left\{u, \tilde{w}_{e}\right\}\right\}-\mathfrak{\Omega}_{\eta}\left[\tilde{w}_{m}\right] .
\end{aligned}
$$

Here, $\{f, g\}=\partial_{u} f \partial_{p} g-\partial_{p} f \partial_{u} g$ denotes the Poisson bracket, while $\tilde{\mathcal{H}} \equiv \mathcal{H}-u_{0} u-\lambda p / 2$. In Eq. (5), we have also added the phenomenological term $\mathfrak{Q}_{\eta}$ accounting for the coupling of the mechanical subsystem to the thermal phonon bath in the leads and other vibrational modes of the nanowire. Based on general consideration regarding quantum dissipative systems [11], it can be written explicitly as $\mathfrak{Q}_{\eta}[w]=\eta\{u, p w\}+D_{\eta}\{u,\{u, w\}\}$, where $\eta=$ $\Omega / Q, Q$ is the quality factor of the mechanical oscillator at zero magnetic field, and $D_{\eta}=\eta / 2 \beta$.

To analyze Eq. (5), we develop a perturbation theory with respect to small $\lambda$ and $\eta$. At zeroth approximation, $\tilde{\mathcal{H}}^{(0)}=\mathcal{H}$ and $\tilde{w}_{m}^{(0)}=W(\mathcal{H})$, where $W$ is an arbitrary function of energy, while $\tilde{w}_{e, \downarrow}^{(0)}$ are defined by the first and second parts of Eq. (5). Substituting $\tilde{w}_{e}$ from the first part of Eq. (5) into the third part of Eq. (5), one gets an equation for $\tilde{w}_{m}^{(1)}$ which is the first order correction to $\tilde{w}_{m}^{(0)}=W(\mathcal{H})$ :

$$
\begin{aligned}
\Omega\left\{\mathcal{H}, \tilde{w}_{m}^{(1)}\right\}= & -\lambda\left(\Omega^{2}-2 \gamma^{2}\right)\left\{u, \tilde{w}_{\downarrow}^{(0)}\right\}-\mathfrak{Q}_{\eta}[W(\mathcal{H})] \\
& -2 \gamma \lambda^{2}\left\{u,\left\{u, W(\mathcal{H})-\gamma \tilde{w}_{\downarrow}^{(0)}\right\}\right\} \\
& -3 \Omega \gamma \lambda\left\{p, \tilde{w}_{\downarrow}^{(0)}\right\}-\Omega\left\{\mathcal{H}-\frac{\lambda}{2} p, W(\mathcal{H})\right\} \\
& +\left\{\tilde{\mathcal{H}}, \mathfrak{R}\left[\tilde{w}_{\downarrow}^{(0)}\right]\right\},
\end{aligned}
$$

$\Re\left[\tilde{w}_{\downarrow}^{(0)}\right] \equiv-2 \Omega \gamma \lambda\left\{u, \tilde{w}_{\downarrow}^{(0)}\right\}+\Omega^{2} \lambda\left\{p, \tilde{w}_{\downarrow}^{(0)}\right\}$. From the first and second parts of Eq. (5), we calculate

$$
\begin{aligned}
\tilde{w}_{\downarrow}^{(0)} & =\Phi(p) W(\mathcal{H}), \\
\Phi(p) & \equiv \frac{1+2 \gamma-2 F(p)}{1+4 \gamma+2 \gamma^{2}-2 F(p)}
\end{aligned}
$$

up to the zeroth order in $\Omega$, enough to preserve the leading contribution in drift and diffusion after insertion into Eq. (6). Expression (7) takes into account the relative shift of $\mu_{S}$ with respect to $\varepsilon_{\uparrow}$ induced by the motion of the nanowire in a magnetic field which generates an electromotive force across the system. Indeed, if $p=0 \Rightarrow$ $F(p)=0$, the population of $\varepsilon_{\uparrow}$ coincides with one for the unmovable nanowire. On the other hand, the motion of the nanowire in a magnetic field with relatively high speed $\left(|p| \gg(\lambda \beta)^{-1} \Rightarrow 2 F(p)= \pm 1\right)$ shifts $\mu_{S}$ well above or below $\varepsilon_{\uparrow}$, depending on the direction of motion, and by this it doubles or annuls a probability of tunneling from source to $\varepsilon_{\uparrow}$. Further, we use the action-angle representation $(2 E, \varphi)$, in which $u=\sqrt{2 E} \cos \varphi, p=\sqrt{2 E} \sin \varphi$, and $\{\mathcal{H}, w\}=\partial_{\varphi} w$, and perform averaging of Eq. (6) over $\varphi$. It annuls the last three terms in Eq. (6), and we obtain a stationary Fokker-Planck equation for $W(E)$ [7] which reduces to

$$
\begin{aligned}
& -\kappa(E) W(E)+D \frac{d}{d E} W(E)=0, \\
& \kappa(E) \equiv-\frac{2 \lambda\left(\Omega^{2}-2 \gamma^{2}\right)}{\sqrt{2 E}}\langle\sin (\varphi) \Phi(\sqrt{2 E} \sin \varphi)\rangle_{\varphi}-\eta,
\end{aligned}
$$

where $\langle\ldots\rangle_{\varphi}$ denotes averaging. The sign of the contribution of the magnetomotive interaction between the nonequilibrium electronic subsystem and the nanowire to the drift coefficient is completely determined by a difference $\Omega^{2}-2 \gamma^{2}$ since $\lambda\langle\sin (\varphi) \Phi(p)\rangle_{\varphi}<0$. Magnetomotive interaction also contributes into the diffusion coefficient, appearing in the second order of $\lambda$, which can be written as $D=\left(4 \gamma \lambda^{2}+\eta \beta^{-1}\right) / 2$ independent of $E$. The solution of Eq. (8) is

$$
W(E)=\mathcal{N} \exp \left(\frac{2 \int_{0}^{E} \kappa\left(E^{\prime}\right) d E^{\prime}}{4 \gamma \lambda^{2}+\eta \beta^{-1}}\right),
$$

where $\mathcal{N}$ is a normalization constant. $W(E)$ describes stationary mechanics of the nanowire in terms of probability in $(u, p)$ phase space dependent on energy. For 
$\kappa(E)<0$, the function $W(E)$ has the only maximum at $E=0$. However, knowing that $\langle\sin (\varphi) \Phi(\sqrt{2 E} \sin \varphi)\rangle_{\varphi} \approx$ $-\gamma \lambda \beta \sqrt{2 E} / 2$ at $E \rightarrow 0$, one can find that $\lim _{E \rightarrow 0} \kappa(E)>0$ if $\gamma \lambda^{2} \beta\left(\Omega^{2}-2 \gamma^{2}\right)>\eta$. In that case, $W(E)$ has a local minimum at $\mathcal{H}=0$, while the maximum is formed at $\mathcal{H}(u, p)=E_{c}$, where $E_{c}$ is a finite zero point of the drift coefficient $\kappa\left(E_{c}\right)=0$ (see Fig. 2). Knowing that $\langle\sin (\varphi) \Phi(p)\rangle_{\varphi}$ saturates to a constant value of the order of $-1 / \pi$ at $E \rightarrow \infty$, it is easy to estimate behavior in the limiting cases. The existence of the maximum of $W(E)$ at energy $E_{c} \neq 0$ corresponds to the formation of a classical limit cycle in the $(u, p)$ phase space of the mechanical system around the unstable static equilibrium point $u, p=0$.

From the above discussion, it appears that the necessary condition for instability to occur is $\omega^{2}-2 \Gamma_{D}^{2}>0$ to ensure that coupling to the nonequilibrium electronic environment results in the pumping of energy into mechanical oscillations. This condition implies that magnetomotive instability starts only if the mechanical frequency is of the same order as the electron tunneling rate to the $D$ lead or higher; otherwise, damping arises. This result is fully consistent with a general statement that in a quasiadiabatic limit, the electromotive interaction generates an additional damping of nanowire vibrations in a system with positive differential conductance. Also, this condition prevents the usage of the rate equation approach [12], utilized in Ref. [5], where the magnetomotive instability in a system with negative DC was studied, and demands a full quantum treatment of the problem presented above. To get the instability, one also needs pumping, generated by magnetomotive interaction with electrons, to overcome the damping coming from the interaction with thermal phonons. It gives the following maximal condition for the zero- $B$ quality factor: $Q^{-1}<\gamma \lambda^{2} \beta\left(\Omega^{2}-2 \gamma^{2}\right) / \Omega$.
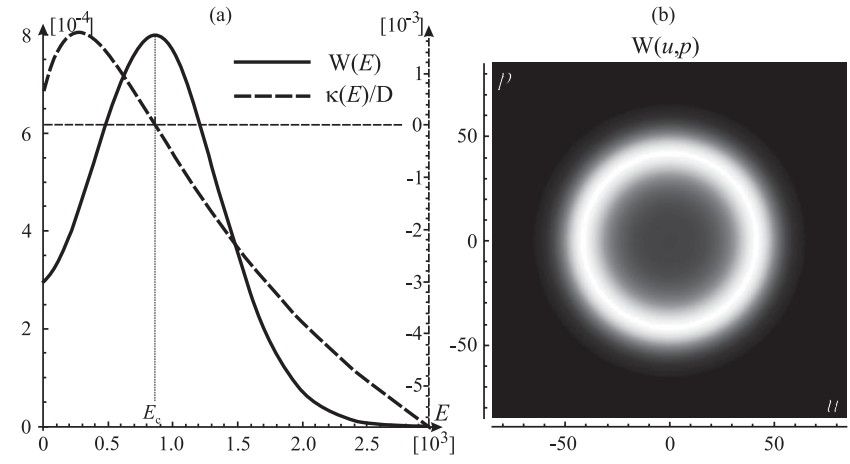

FIG. 2. (a) The "dynamical potential" $\kappa(E) / D$ whose zero point $E_{c}$ determines the maximum of the Wigner function $W(E)$ describing the probability of finding a mechanical system at a certain energy $E$ (dimensionless units). (b) Density plot of the probability $W(u, p)$ in mechanical $(u, p)$ phase space, indicating the existence of a classical limit cycle at its maximum measured from the static equilibrium state determined by $u_{0}$. The used parameters are $\lambda=0.1, \gamma=0.1, \Omega=0.3, \beta=0.95$, and $\eta=3.5 \times 10^{-5}\left(Q=8.6 \times 10^{4}\right)$.
Taking the small parameters $\Omega, \lambda, \gamma \sim 10^{-1}$ and $\beta \sim 1$ $(L \sim 1 \mu \mathrm{m}$, nanotube diameter $d \sim 1 \mathrm{~nm}, \hbar \omega \sim 1 \mu \mathrm{eV}$, $\hbar \Gamma_{S} \gtrsim 1 \mu \mathrm{eV}, T \sim 10 \mathrm{mK}, B \sim 10 \mathrm{~T}$ ), we find that $Q$ should be greater than $10^{4}$ to obtain an oscillation amplitude of a few nanometers. This is well within the possible experimental setup [7,13].

The physical picture: The event of tunneling of an electron from an $S$ lead to a nanowire and from a nanowire to a $D$ lead instantly changes the mechanical momentum of the nanowire by $\Delta p=\xi e B L / c$. If an average value of $\dot{u} \Delta p$ is positive, the pumping of mechanical energy arises. Therefore, the instability may be achieved by the proper correlation between the tunneling events and the direction of the nanowire velocity. Here, such correlations are induced by the motion of the nanowire in a magnetic field and are arranged by an interplay between specific biasing [Fig. 1(b)] and the Coulomb blockade effect. If one ignores the electromotive force, an electron, after tunneling from an $S$ lead to the $\varepsilon_{\uparrow}$ state on a nanowire, will most likely tunnel back, since $\Gamma_{S} \gg \Gamma_{D}$, thus producing random momentum transfer $\pm \Delta p$ leading to diffusion in the phase space. On the other hand, the electron in the $\varepsilon_{\downarrow}$ state cannot tunnel back to the $S$ lead, due to the lack of empty states at accessible energies, and always transfers momentum $+\Delta p$. The motion of the nanowire in a magnetic field generates the electromotive force $\xi B L \dot{u}$, depending on the direction of velocity, that adds to the bias voltage. It changes the effective chemical potential in the leads, shifting $\mu_{S}$ above $\varepsilon_{\uparrow}$ if $B \dot{u} \propto \dot{u} \Delta p>0$, or below $\varepsilon_{\uparrow}$ if $B \dot{u} \propto \dot{u} \Delta p<0$. At the same time, the Coulomb blockade effect, prohibiting the double occupancy of the nanowire, induces correlation between the relative position of the electrochemical potential and events of tunneling to the $\varepsilon_{\downarrow}$ state. If $\mu_{S}<\varepsilon_{\uparrow}$, the occupation of the $\varepsilon_{\uparrow}$ state is reduced and tunneling to the $\varepsilon_{\downarrow}$ state will more likely take place during this phase of oscillation. It corresponds to $\dot{u} \Delta p>0$; thus, charge transfer through the $\varepsilon_{\mid}$state results in energy pumping to the mechanical subsystem, while the transport through the $\varepsilon_{\uparrow}$ state leads to a dissipation of mechanical energy. Indeed, in a phase of oscillations when $\dot{u} \Delta p<0$, the electrochemical potential shifts above $\varepsilon_{\uparrow}$, thus increasing the probability of tunneling to the $\varepsilon_{\uparrow}$ state and then to the $D$ lead. If it occurs while the nanowire continues to move in that direction, which definitely happens if $\Gamma_{D} \gg \omega$, it results in an energy loss. The necessary condition for the instability $\omega \gtrsim \Gamma_{D}$ implies, in fact, a requirement that the nanowire change its direction of motion before the electron tunnels from the $\varepsilon_{\uparrow}$ state to the $D$ lead. As a result, the pumping may overcome total damping and the instability arises. In the high energy limit $(\lambda \beta \sqrt{E} \gg 1)$, the amplitude of oscillations becomes large, generating the electromotive force that shifts $\mu_{S}$ outside the pumping-active interval within the $k_{B} T$ vicinity around $\varepsilon_{\uparrow}$. As the amplitude grows, the system spends less and less time in the pumping-active interval, effectively leading to an internal saturation of pumping. 
In conclusion, we have investigated the electromechanical stability of the nanowire, exposed to the external magnetic field and suspended to two normal-metal leads under dc bias with essentially positive differential conductance. We show that the tunneling through two spin channels with different energies and correlated by the Coulomb blockade leads to the instability and an onset of self-excited oscillations in transport and mechanical vibrations if the retardation effects, coming from the mechanical subsystem and coupled by electromotive coupling, are pronounced enough. Since the mechanism of achieving the instability is based on the effective gating effect by the occupation of a higher spin state that synchronizes the tunneling process and momentum transfer into a mechanical subsystem with the particular direction of motion of the nanowire, in the setup with positive differential conductance, it is essential that mechanical vibrations have frequency of the same order or higher than the electron tunneling rate to the drain lead in order to maintain the coherent momentum transfer. The instability appears as soon as the energy pumped into the system exceeds the mechanical dissipation, which can be made small enough in modern manufactured carbon nanotubes. Our numerical analysis indicates that the instability leads to sustained oscillations with an amplitude of a few nanometers. Such are detectable by modern experimental techniques [14].

Financial support from the Croatian Ministry of Science, Education, and Sports (Project No. 119-1191458-1023) and the Swedish Research Council (VR) is greatly acknowledged. The authors are grateful to Y. V. Nazarov for fruitful discussions.

*Corresponding author. dradic@phy.hr

[1] A. N. Cleland, Foundations of Nanomechanics: From Solid-State Theory to Device Applications (Springer, Berlin, 2003).

[2] S. Sapmaz, Ya. M. Blanter, L. Gurevich, and H. S. J. van der Zant, Phys. Rev. B 67, 235414 (2003); L. M. Jonsson, L. Y. Gorelik, R. I. Shekhter, and M. Jonson, Nano Lett. 5, 1165 (2005); A. K. Hüttel, B. Witkamp, M. Leijnse, M. R.
Wegewijs, and H. S. J. van der Zant, Phys. Rev. Lett. 102, 225501 (2009); S. Braig and K. Flensberg, Phys. Rev. B 68, 205324 (2003); J. O. Island, V. Tayari, A. C. McRae, and A. R. Champagne, Nano Lett. 12, 4564 (2012); B. Lassagne, Y. Tarakanov, J. Kinaret, D. Garcia-Sanchez, and A. Bachtold, Science 325, 1107 (2009).

[3] L. Y. Gorelik, A. Isacsson, M. V. Voinova, B. Kasemo, R. I. Shekhter, and M. Jonson, Phys. Rev. Lett. 80, 4526 (1998).

[4] Ya. M. Blanter, O. Usmani, and Yu. V. Nazarov, Phys. Rev. Lett. 93, 136802 (2004); 94, 049904(E) (2005).

[5] D. Radić, A. Nordenfelt, A. M. Kadigrobov, R. I. Shekhter, M. Jonson, and L. Y. Gorelik, Phys. Rev. Lett. 107, 236802 (2011).

[6] R. I. Shekhter, L. Y. Gorelik, L. I. Glazman, and M. Jonson, Phys. Rev. Lett. 97, 156801 (2006).

[7] See Supplemental Material at http://link.aps.org/ supplemental/10.1103/PhysRevLett.111.186802 for the detailed derivation of model Hamiltonian, Liouville-von Neumann equation, the collision integral, as well as the discussion on an optimization of system parameters for experimental setups.

[8] L.D. Landau and E. M. Lifshitz, Theory of Elasticity (Pergamon, Oxford, England, 1970), 2nd ed., Vol. 7.

[9] T. Novotny, A. Donarini, and A.-P. Jauho, Phys. Rev. Lett. 90, 256801 (2003); D. Fedorets, L. Y. Gorelik, R. I. Shekhter, and M. Jonson, Phys. Rev. Lett. 92, 166801 (2004).

[10] A. Zazunov, D. Feinberg, and T. Martin, Phys. Rev. B 73, 115405 (2006).

[11] H.P. Breuer and F. Petruccione, The Theory of Open Quantum Systems (Oxford University Press, New York, 2002).

[12] The rate equation approach is justified only if a proper average over many electron tunnelings can be taken during one period of mechanical vibration. Here, it is not the case due to the minimal instability condition.

[13] A. K. Hüttel, G. A. Steele, B. Witkamp, M. Poot, L. P. Kouwenhoven, and H. S. J. van der Zant, Nano Lett. 9, 2547 (2009); G. A. Steele, A. K. Hüttel, B. Witkamp, M. Poot, H. B. Meerwaldt, L. P. Kouwenhoven, and H. S. J. van der Zant, Science 325, 1103 (2009).

[14] D. Garcia-Sanchez, A. San Paulo, M. J. Esplandiu, F. Perez-Murano, L. Forró, A. Aguasca, and A. Bachtold, Phys. Rev. Lett. 99, 085501 (2007); M. Lexholm, I. Karlsson, F. Boxberg, and D. Hessman, Appl. Phys. Lett. 95, 113103 (2009). 